\title{
CONSTRUCTION AND VALIDATION OF SMALL ELECTRO-MECHANICAL VIBRATION SENSORS
}

\author{
ADOLFO SABATO \& ALESSANDRO SABATO \\ Laboratory of TCA, University of Calabria, Rende (CS), Italy.
}

\begin{abstract}
This project offers the opportunity to investigate oscillatory phenomena that can jeopardize the safety of structures of particular importance, using the accelerometers installed on small electromechanical $(M E M S)$ devices known as Motes, which are devices that transmit data remotely without the need of using cable connections. The term 'Motes' usually indicates the single node in a Wireless Sensor Network (WSN). Using the accelerometer sensor installed on these tools, it is possible to program a network with numerous sensors to monitor the behavior of the system being tested.

The present state of research has made it possible to obtain a prototype sensor by means of which data can be compared with those measured using traditional systems to detect accelerations (piezoelectric accelerometers). The first step was that of defining the calibration procedure. Once the calibration curve has been completed in the laboratory, the single node (Mote) can be programmed for the acquisition of a single physical quantity and can also be scheduled to transmit these values to create a WSN, comprising numerous sensors connected to a central node that acts as a receiver.

All programming is done using the nesC language, and it is finally loaded onto the sensor using the TinyOS operating system for the management of the nodes.

During the measurements, one of the main problems manifested by these devices was the discharge of the batteries after a prolonged use. This is highly interesting because the 'offset value' characteristic of the sensor is influenced by the energy possessed by the batteries.

Another problem highlighted by the tests conducted is the low sensitivity of the accelerometers, compared to that of a seismic accelerometer, a feature causing the loss of events with an intensity below a certain threshold.

Nevertheless, these systems make it possible to monitor, with very low costs, critical structures that are subject to physical events of particular intensity to prevent disaster.

Keywords: Experimental analysis, MEMS accelerometers, motes, vibration.
\end{abstract}

\section{INTRODUCTION}

The generation of vibrations is characteristic of a multitude of operational processes. We can consider, for example, both the functioning of equipment and the transit of vehicles over infrastructures, such as bridges and railway lines. Such vehicles not only produce phenomena of noise pollution but can also be the cause of damage and even destruction to structures and buildings. It is therefore necessary to plan campaigns for their measurement. To do so, we use accelerometers that, depending on their fields of application and accuracy of measurement, can be of different types, even though they have the common characteristic of having high costs and the need for the measuring device to be positioned close to the tested system, since the length of wirings connecting the sensor to the analyzer cannot exceed certain limits.

For this reason, we are seeking to realize devices that are small and able to send sampled data through wireless technology, freeing technicians from using wirings and other instrumentations for signal transmission. The present work aims at shaping the accelerometers installed on Micro Electro-Mechanical System (MEMS) devices, also known as Motes. With such a term, usually, the single knot of a Wireless Sensor Network (WSN) [1] is indicated, by means of which it is possible to manage a network composed of several sensors [2] able to observe the behavior of the system being tested and able to transmit sampled data without using cables, the form of instrumentation which is usually used. 


\section{PROTOTYPE IMPLEMENTATION AND CALIBRATION}

To do so, during the study, some motes, produced by the American company Crossbow, were used. In particular, the model named MPR400 (commercially known as MICA2) was employed. The sensor boards associated to that device were those of the MTS310 series with a biaxial accelerometer ADXL202JE inside, while as output knot for the network and gateway the MIB510 system was used [3]. The state-of-the-art model reached by this technology enabled the realization in the Laboratory of a prototype that compares the sampled data and that measured using traditional accelerometers (in this case, seismic piezoelectric accelerometers AP2050 by AP Tech).

Figure 1 which follows shows, in detail, a part of the instrumentation used and, in particular, the sensor board on which the accelerometer is installed.

These devices are powered by two $1.5 \mathrm{~V}$ batteries, while the data transmission occurs through radio equipment made by an antenna. These characteristics show how these instruments can be utilized to affect a remote switch control of the area without the necessity of positioning an analyzer close to the site.

Before going on with a measurement campaign with the instrumentation described above, a coding operation has been necessary. The management system of these devices is made of an operative system called TinyOS and fitting routines written in nesC [4]. By means of this operation, it was possible to enable the functioning of the accelerometer placed over each sensor board and also to identify it, through an ID number, in the network. The first operation was necessary so as to avoid energy waste connected to the use of the sensors installed (these devices are provided with a biaxial magnetometer, a thermometer and a luxmeter, and, obviously, the contemporary use of all these devices causes a rapid depletion of the batteries). The second operation, on the other hand, identifies the mote in the network and fixes a transmission frequency.

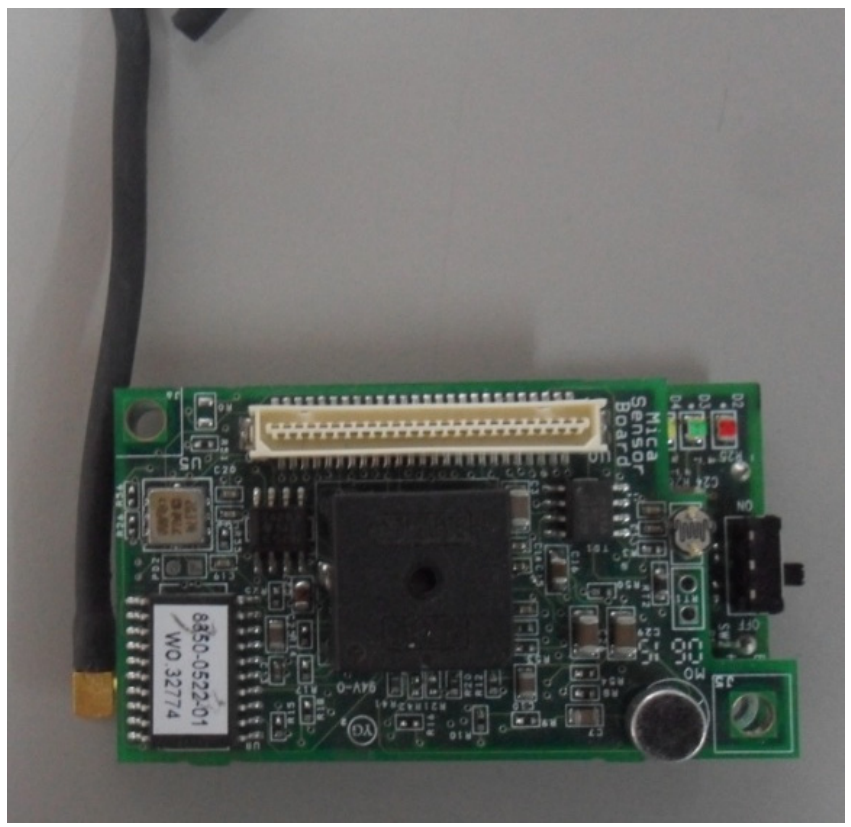

Figure 1: Sensor board MTS310 used for acceleration measurements. 
The ADXL202JE accelerometer installed over these motes is a biaxial sensor, able to measure both positive and negative accelerations up to a maximum level of $\pm 2 g$. Provided with integrated circuits, it converts the sampled analogical signal into a digital one as a rectangular wave, whose duty cycle will be proportional to the measured acceleration. This is possible because the accelerometer is formed by a variable condenser, constituted, afterward, by a plate attached to a vibrating mass between two immobile armatures. Every stress phenomenon will cause a shifting of the mass causing a variation in its capacity and consequently a potential difference from that which primarily characterized the rest conditions. This variation will produce the rectangular wave that is proportional to the acceleration experienced.

During this study, two operations were conducted: one regarding the evaluation of the offset values (i.e. the value that corresponds to the absence of stress) characteristic of the instrumentation and the second regarding how the depletion of the batteries can affect the measured data.

All these operations were determined by considering how these sensors work. In effect, accelerometers, as mentioned before, to supply the acceleration data, utilize the potential difference value that is produced in the condenser due to the variation of its capacity caused by the moving plate during the stresses. It is, therefore, very important to evaluate these values correctly because they are the principal parameters and may vary depending on remaining battery life and other parameters (such as temperature), which are related to the type of operating environment.

The first operation was necessary since the different models employed, in spite of their all belonging to the same series, gave, in a state of rest, values of acceleration nonzero and those different from each other. In these situations, as it is possible to verify by using traditional accelerometers, it would be logical to expect values along the two axes $X$ and $Y$, taking into account the sensitivity of the device, close to the value $\mathrm{a}=0 \mathrm{~g}$, if the sensor is positioned perpendicularly to the Earth's gravitational field; or equal to the value $\mathrm{a}= \pm 1 \mathrm{~g}$ when the sensor is set out with one of its axes oriented along the direction of the acceleration of gravity vector. To sum up, for the different series of utilized sensors, the potential differential values corresponding to the state of rest were characterized.

To do this, a calibration procedure was planned, based on the characterization of wellknown angles and exploiting the potentiality of the board as static sensor for the detection of the tilt angles. Knowing, in fact, the intensity of these points, it was possible to make a numerical interpolation that allowed one to determine the mathematical relationship between these values. Effectively, a calibration curve was made, orienting the board along different directions, to subject the axes of the sensor to a constant acceleration equal to $1 \mathrm{~g}$ (and of course equal to $0 \mathrm{~g}$ in the direction perpendicular to it) and measure the value given by the mote and then correlate it with those well known of $0 \mathrm{~g}$ and $\pm 1 \mathrm{~g}$.

In this case, the sensors are connected to the MIB510 gateway and so fed by mains electricity, with a system able to ensure a constant voltage power supply so as to obtain a stable data, not affected by the residual battery charge.

The sensor is placed on a metallic support in compliance with the ISO 9913 and ISO 9614 standards and laid over a position maintained horizontal and at rest.

Sensors are placed in five different positions (shown by capital letters from 'A' to 'E') to subject the axes of the board, alternately, to well-known accelerations and they, therefore, measure the values given by the mote. In the example shown here, we have the test given for the sensor called MTS310\#1 in the position indicated as 'A', or with both axes perpendicular to the vector acceleration of gravity. 
This position, maintaining the mote in a horizontal position, and therefore with both axes perpendicular to the $\mathrm{G}$ vector, allowed us to evaluate the offset value characteristic of the sensor. The other four, on the other hand, orienting the gateway with an axis, in turn, parallel to the lines of force of the gravitational field, allowed us to estimate the values equivalent to those fixed as well known of $\pm 1 \mathrm{~g}$ and consequently the orientation, both positive and negative, of the axes.

Each measurement was 2 min long with sampling time of the signal equal to $125 \mathrm{~ms}$. This allowed for the recording of a number of samples large enough to carry out a significant statistical analysis. The sampled data recorded by this sensor is shown below in Table 1.

As highlighted in Table 1, for each axis two positions exist for which the measured values of acceleration remain constant and two other positions for which the difference between the values is maximum. These last two quantities, and the position associated to them, indicate the values corresponding to those well known of $\pm 1 \mathrm{~g}$ and the directions for which the instrumentation is oriented in the same direction of gravity acceleration. In particular, considering the values of Table 1, it is possible to affirm that the accelerometer mounted in the sensor named as MTS310\#1 measures, along the $X$ axis, acceleration values identical in the position named as ' $\mathrm{D}$ ' and ' $\mathrm{E}$ ', and values for which maximum shifting $\Delta x$ is in the positions called ' $\mathrm{B}$ ' and ' $\mathrm{C}$ '. This means that, disposing the gateway along the ' $\mathrm{B}$ ' and ' $\mathrm{C}$ ' direction, which is vertical, you orient the instrument parallel to the gravitational field and therefore you measure its maximum value $(C)$ and the other its minimum value (B) once. It is possible to follow the same reasoning along the $Y$ axis. In fact, positions ' $\mathrm{B}$ ' and ' $C$ ' record the same values of acceleration, while the positions ' $D$ ' and ' $E$ ' measure the wide shifting $\Delta y$. Once these values are known, it is possible to determine the offset value characteristic for the accelerometer. For example, one can consider the acceleration recorded along the $Y$ axis in the positions 'D' and 'E', equal to $60 \mathrm{mg}$ and to $2340 \mathrm{mg}$. These can be matched, respectively, with the values of $-1 g$ and $+1 g$; it seems obvious that the value equal to $0 \mathrm{~g}$, that is, the offset value, should be the midpoint between and equal to $1200 \pm 20 \mathrm{mg}$, which is exactly equal to that measured in the 'A' position. It is possible to apply the same argument with the accelerations recorded along the $X$ axis in the positions ' $B$ ' and ' $C$ ', obtaining a midpoint equal to $960 \pm 20 \mathrm{mg}$, once more coinciding with that measured in the position shown in Fig. 2. To conclude, it is possible to affirm that the offset values are equal to those recorded maintaining the accelerometer with both axes perpendicular to the lines of force of the gravitational field, which is really embodied by the position 'A'.

Afterwards, for each sensor it will be necessary to consider as zero value the one calculated before and, based on these values, to correct the instantaneous values derived from measures.

Table 1: Values of acceleration sampled by mote MTS310\#1.

\begin{tabular}{lrrrrr}
\hline & \multicolumn{5}{c}{ Position } \\
\cline { 2 - 6 } & A & B & C & \multicolumn{1}{c}{ D } & \multicolumn{1}{c}{ E } \\
\hline$x(m g)$ & 960 & -180 & 2080 & 940 & 960 \\
$y(m g)$ & 1200 & 1200 & 1200 & 60 & 2340 \\
$V(m V)$ & 3295 & 3295 & 3295 & 3295 & 3295 \\
\hline
\end{tabular}




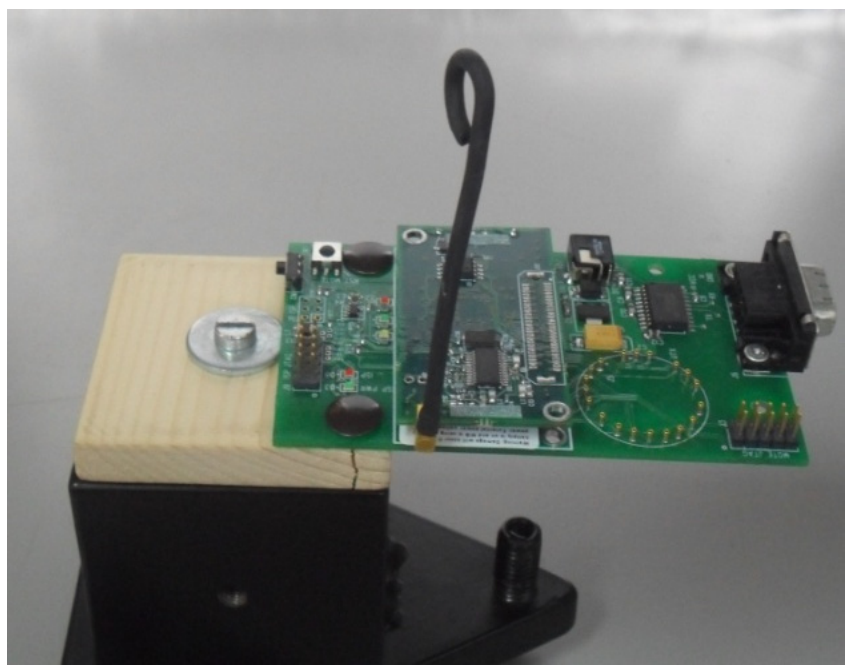

Figure 2: Sensor board placed horizontally in position 'A'.

Based on the offset values and on the width of the interval $\Delta x$ e $\Delta y$ calculated for each device, the following general relation was determined:

$$
\left\{\begin{array}{l}
x_{c o r r, t}=\frac{\left(x_{0}-x_{m i s, t}\right)}{\Delta x / 2} \\
y_{c o r r, t}=\frac{\left(y_{0}-y_{m i s, t}\right)}{\Delta y / 2}
\end{array}\right.
$$

where the terms $x_{0}$ and $y_{0}$ stand for the offset values characteristic for each sensor, the terms $x_{\text {mis, }}$ and $y_{\text {mis, }}$ stand for the values of acceleration measured by the sensor at the generic temporal instant $t, x_{c o r r, t}$ and $y_{c o r r, t}$ stand for the values correct, always at the generic temporal instant $t$, while $\Delta x$ and $\Delta y$ stand for the shifting characteristic for each sensor.

It is very interesting to study the trend indicated by the offset values when the supply power changes, to verify whether the progressive exhaustion of the battery could significantly damage or change the measurement.

\section{PRELIMINARY ASSESSMENTS FOR MOTES USE}

As has already been mentioned, one of the main faults of the generation of motes and sensors used in this study is represented by the waste of energy that marks the data transmission. This consumption, obviously, restricts the life of the batteries, so the second test was necessary to estimate how those values used as offset were influenced by the remaining charge of the cell power. To do this, the sensors were maintained at rest on the same support shown before and left to discharge, while acceleration values, and corresponding tension values, were recorded up to the value of $1900 \mathrm{mV}$ that represents the functioning limit for the devices. The aim of this test is that of obtaining a simple qualitative description of the relation between the supply power of the batteries and the measured values of acceleration. As shown in Fig. 3, this value decreases in time and afterward with decreasing battery life. 


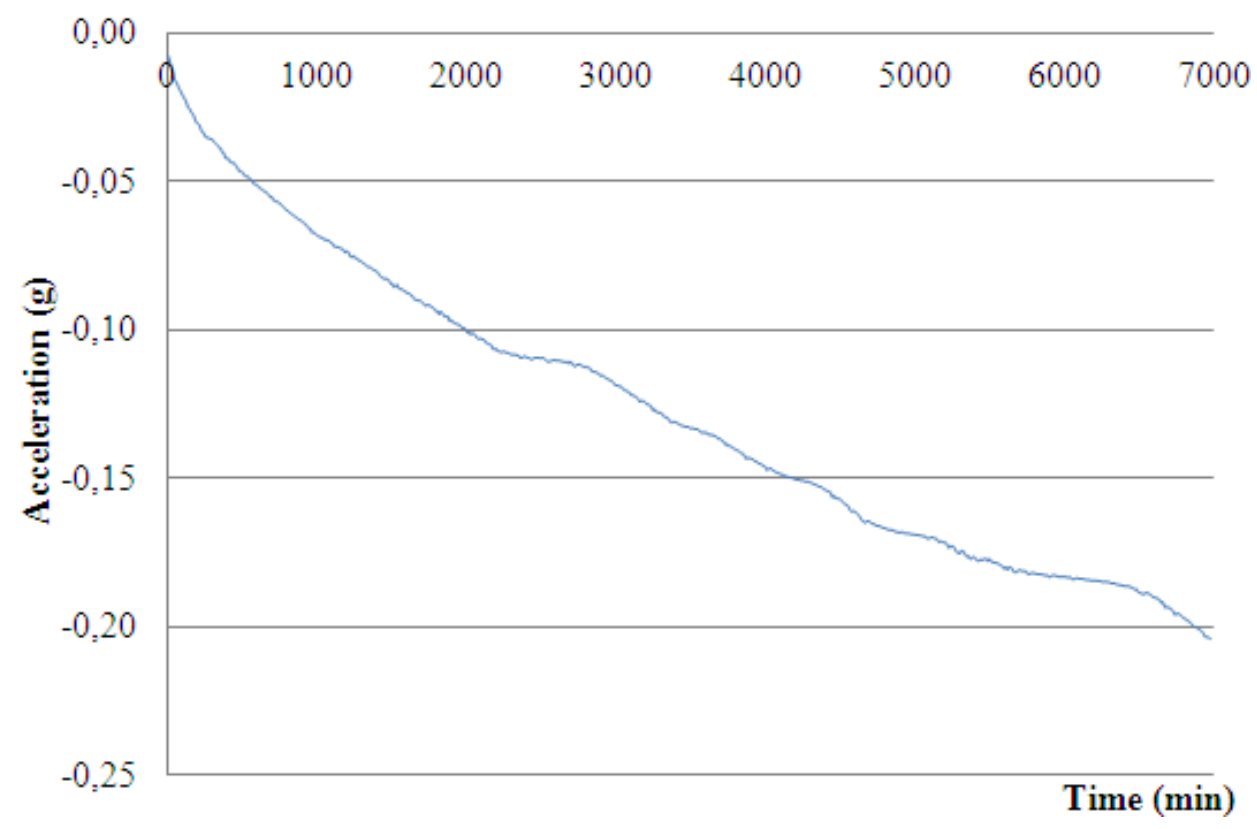

Figure 3: Variation in offset value due to variation of supplied voltage.

The sampling time is always equal to $125 \mathrm{~ms}$, equal to sending eight data packs in a second. Nevertheless, it is possible to observe, analyzing the sequence of received data, how for supply values inferior to $1950 \mathrm{mV}$, this number is always smaller, and a few instants before batteries reach the point of exhaustion, transmitted data is sent over longer intervals of time. The following figure, Fig. 3, shows the trend of the offset values for the sensor in time.

The last problem, highlighted by these tests is with regard to the sensitivity of accelerometers mounted over the boards. In fact, this, compared with the sensitivity of traditional accelerometers (seismic piezoelectric) is an order of lower magnitude, and this causes a loss of information about events having intensity below a certain threshold.

This is highlighted by Fig. 4 in which a signal regarding the transit of a vehicle over a metallic girder bridge [5], recorded by traditional accelerometers and MEMS accelerometers, is shown.

Observing the signal obtained with the traditional accelerometer (at the bottom of the image), it is possible to note how even the smallest perturbation was highlighted and that the vehicular transit close to the measuring device appears clearly; while looking at the part of the figure regarding the signal sampled with the mote, it is possible to observe a variable signal, fluctuating between two values, zero and a value equal to the sensitivity of the device. This does not mean that the system suffers accelerations equal to, in the examined case, $0.17 \mathrm{~ms}^{-2}$ but rather that the scale of the device sustains some small variation and therefore it 'reads' the value by zero-turns allowing for the sensitivity of the device. With regard to the transit, it is characterized by a peak having intensity equal to $0.37 \mathrm{~ms}^{-2}$. One can observe how the sensor installed over the mote, even if it describes the phenomenon in a less detailed manner (particularly with regard to its exhaustion), allows one to estimate the value of acceleration $\left(0.34 \mathrm{~ms}^{-2}\right)$, an assessment, however, very close to that made by traditional instruments. 


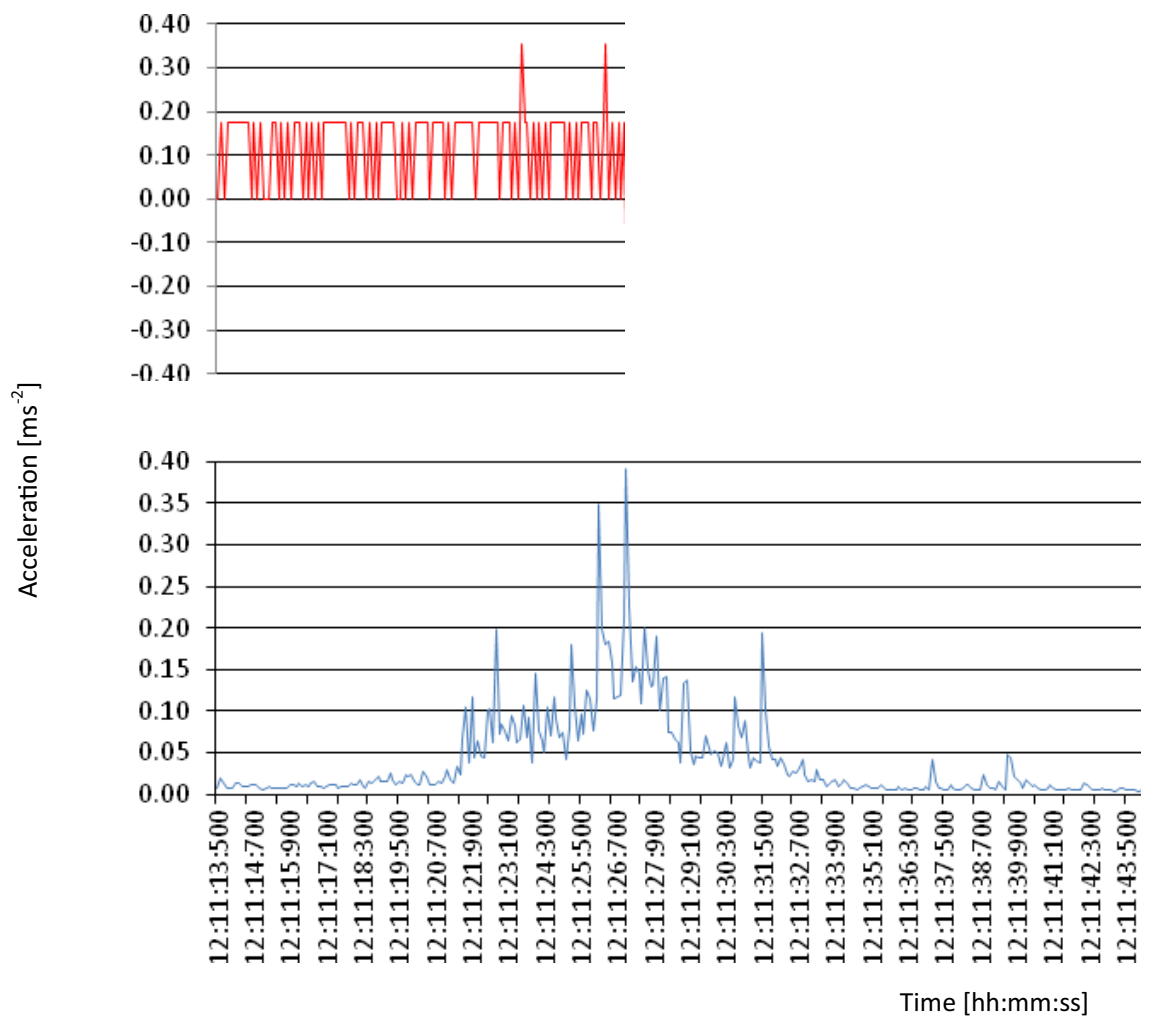

Figure 4: Comparison between the time history measured with the mote MTS310\#1 and the seismic accelerometer.

\section{CONCLUSION}

To conclude, it is possible to affirm that the technology that equips motes is still far from achieving the quality standards that are typical of the instrumentation in accordance with international standards used to validate measurements. Nevertheless, if one is not interested in quantitative analysis but only in studying the phenomenon from a qualitative point of view, these tools perform this task perfectly. Furthermore, the low sensitivity of this type of equipment does not constitute an excessive application limit in the field of structural health, because stress capable of damaging structural and mechanical elements exceeds these values. These systems make it possible to keep a close watch, with very low costs, on critical structures which are subject to physical events of particular intensity to prevent disaster with regard to the stability of the structure observed. In effect these devices cost about one-tenth of the traditional ones. Therefore, it is possible, at the same cost, to exert control over several points to ensure a more careful analysis in the protection of the monitored system. Once again, we wish to underline the possibility of raising the level of environmental safety through the commitment of modest amounts of financial resources. For example, through constant monitoring, it will be possible to control the type of stresses generated by the passage of particular types of vehicles on sensitive targets, such as large bridges, and, in an emergency, ban vehicular passage over it, to assess the response of buildings and their health during the occurrence of events of significant intensity, or even monitor the health of pieces of equipment subject to periodic stress, such as train axles, thus preventing them from breaking. 


\section{REFERENCES}

[1] Zhao, F. \& Guibas, L., Wireless Sensor Networks - An Information Processing Approach, Morgan Kaufmann: San Francisco, CA, USA, 2004.

[2] Banatre, M., Marron, P.J. \& Ollero, A., Cooperating Embedded Systems and Wireless Sensor Networks, Wiley-Iste: Hoboken, NJ, USA, 2008.

[3] Crossbow technology, Wireless Sensor Networks, Getting Started Guide - Rev. A, available at http://www.xbow.jp/telos_guide_7430-0380-01_a.pdf, 2005 (accessed March 2009)

[4] Kernighan, B.W. \& Ritchie, D.M., The C Programming Language, 2nd edn., Prentice Hall: Upper Saddle River, NJ, USA, 1988.

[5] UNI 10985, Vibrations on bridges and viaducts - General guidelines for the execution of dynamic tests and investigations, 2002. 